\title{
Recent Advances in the Neural Control of Movements: Lessons for Functional Recovery
}

\author{
Mark L. LAtAsh, Ph.D. ${ }^{1}$ and Momoko YamagATA, Ph.D. ${ }^{2,3,4}$ \\ ${ }^{1)}$ Department of Kinesiology, The Pennsylvania State University, USA \\ ${ }^{2)}$ Department of Human Development, Graduate School of Human Development and Environment, Kobe University, Japan \\ ${ }^{3}$ Department of Physical Therapy, Human Health Science, Graduate School of Medicine, Kyoto University, Japan \\ ${ }^{4)}$ Research Fellow of the Japan Society for the Promotion of Science, Japan
}

\begin{abstract}
We review the current views on the control and coordination of movements following the traditions set by Nikolai Bernstein. In particular, we focus on the theory of neural control of effectors - from motor units to individual muscles, to joints, limbs, and to the whole body - with spatial referent coordinates organized into a hierarchy with multiple few-to-many mappings. Further, we discuss synergies ensuring stability of natural human movements within the uncontrolled manifold hypothesis. Synergies are organized within the neural control hierarchy based on the principle of motor abundance. Movement disorders are discussed as consequences of an inability to use the whole range of changes in referent coordinates (as in spasticity) and an inability to ensure controlled stability of salient variables as reflected in indices of multielement synergies and their adjustments in preparation to actions (as in brain disorders, including Parkinson's disease, multiple-system atrophy, and stroke). At the end of the review, we discuss possible implications of this theoretical approach to peripheral disorders and their rehabilitations using, as an example, osteoarthritis. In particular, "joint stiffening” is viewed as a maladaptive strategy, which can compromise stability of salient variables during walking.
\end{abstract}

Key words: Synergy, Stability, Agility, Referent coordinate, Osteoarthritis

(Phys Ther Res 00: 00-00, 0000)

\begin{abstract}
The fields of the neural control of movements and movement disorders have always been tightly intertwined. Indeed, observations of neurological patients have led to a number of influential concepts such as those of hierarchical control of movements, multiple cortical representations, and synergy ${ }^{1,2)}$. The highly influential theoretical views of Nikolai Bernstein ${ }^{3,4)}$ have been largely based on observations of patients with a variety of motor disorders and also produced important insights for motor rehabilitation. In particular, Bernstein emphasized the importance of plastic changes within the central nervous system leading to adaptive adjustments in movement patterns.
\end{abstract}

Received: May 4, 2021

Accepted: July 12, 2021

Advance Publication by J-STAGE: September 29, 2021

Correspondence to: Mark L. Latash, Department of Kinesiology, The Pennsylvania State University, USA, University Park, PA, 16802, USA

\# e-mail: mll11@psu.edu

doi: 10.1298/ptr.R0018
These insights have important implications for a number of issues related to practice of motor rehabilitation. These include the following: Should the goal of therapy be bringing the movement patterns of a patient back as close as possible to those observed in healthy persons ("normal movements")? Should therapy emphasize functional recovery even at the expense of apparently abnormal movement patterns being used to solve everyday motor problems? In cases of a localized injury, should therapy be focused on that locus or involve the whole body?

Discussions of these issues have been ongoing over the past years with the accumulation of knowledge about the neural mechanisms of the production of voluntary movements ${ }^{5-7)}$. Indeed, the importance of basic knowledge for motor rehabilitation has become obvious for both researchers and practitioners. Hence, the main purpose of this review is to present an update on the current understanding of the neural mechanisms involved in the production (and, sometimes, perception) of human voluntary movements with an emphasis on most recent developments. Of course, 

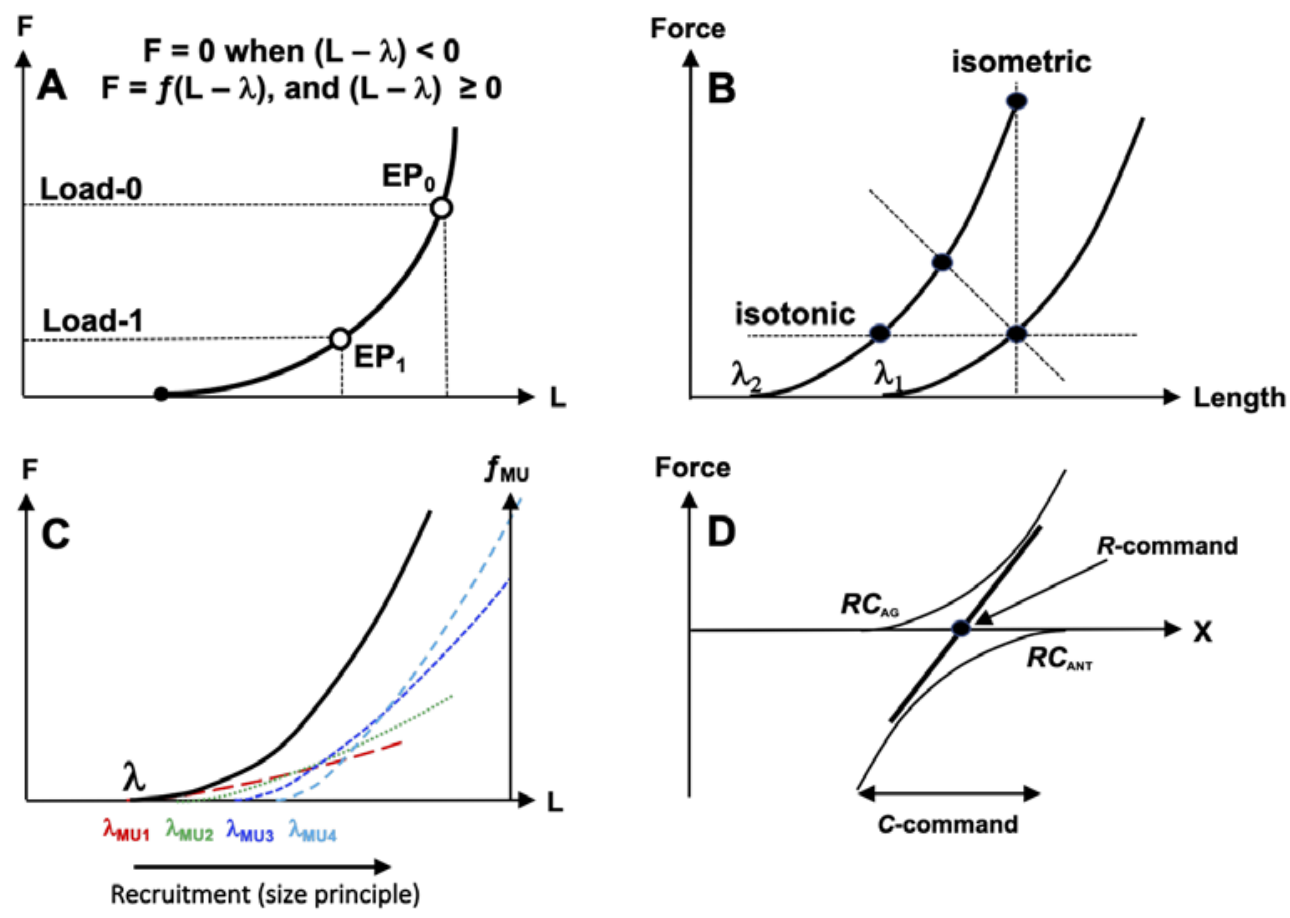

Fig. 1

A: Setting a value of stretch reflex threshold, $\lambda$ produces a dependence of muscle active force on muscle length, $F(L)$. A change in external load leads to movement from the initial equilibrium point $\left(\mathrm{EP}_{0}\right)$ to another one, $\mathrm{EP}_{1}$. B: The production of voluntary movements is associated with changes in $\lambda$, for example from $\lambda_{1}$ to $\lambda_{2}$. The same shift of $\lambda$ can lead to different mechanical effects depending on the external load. C: Each motor units (MU) can be described with a corresponding $\lambda$ value resulting in a dependence of its firing frequency $(f)$ on muscle length. D: The control of an effector can be described with time-varying referent coordinates $\mathrm{RC}_{\mathrm{AG}}$ and $\mathrm{RC}_{\mathrm{ANT}}$ (for the agonist and antagonist muscles). Alternatively, $R$-command (reciprocal command) and $C$-command (coactivation command) can be used.

this review reflects the subjective opinions of the authors and may not be shared by all of our respected colleagues.

We accept, as an axiom, that the human body, including the brain, is a product of natural evolution and, as such, it has to obey laws of nature ${ }^{8,9)}$. Some of those laws are described in physics textbooks. They are common across the inanimate world and biological objects. These laws are very powerful in predicting behavior of inanimate objects, but not so - for biological objects. Indeed, they only constrain biological behaviors but do not prescribe them. As a result, biological objects, including humans, commonly show movements, which are not observed in the inanimate world. Obvious examples include walking uphill and swimming against the current. This means that some of the laws of nature are specific to biological objects, and we see discovering such biology-specific laws as imperative for progress in the field of the neural mechanisms of behavior.

We begin this review with a brief exposition of the theory of motor control with time-varying spatial referent coordinates for the effectors $^{8,10)}$, explain how this theory can be applied to objects ranging from motor units to the whole body, and then describe recent applications of this theory to clinical issues such as spasticity and its treatment. Further, we shift emphasis to the issue of movement stability and introduce the principle of abundance, the notion of performance-stabilizing synergies, and the toolbox associated with the uncontrolled manifold hypothesis ${ }^{11-13)}$. We review recent studies documenting changes in the ability to control stability of movements in task-specific manner in a variety of neurological patients. At the end, we consider osteoarthritis as a pathological condition, which has not been discussed within the introduced theoretical frameworks.

\section{Control of Biological Movement with Referent Coordinates}

The theory of motor control with spatial referent coordinates (RCs) originates from the classical equilibriumpoint hypothesis (EP-hypothesis) ${ }^{14,15)}$. According to the EPhypothesis, the neural control of a muscle with its intact connections to the spinal cord can be adequately described as setting time changes in the threshold of the stretch reflex, $\lambda$. Note that setting a value of $\lambda$ produces a dependence of muscle active force on muscle length, $F(\mathrm{~L})$ (Fig. 1 A), 


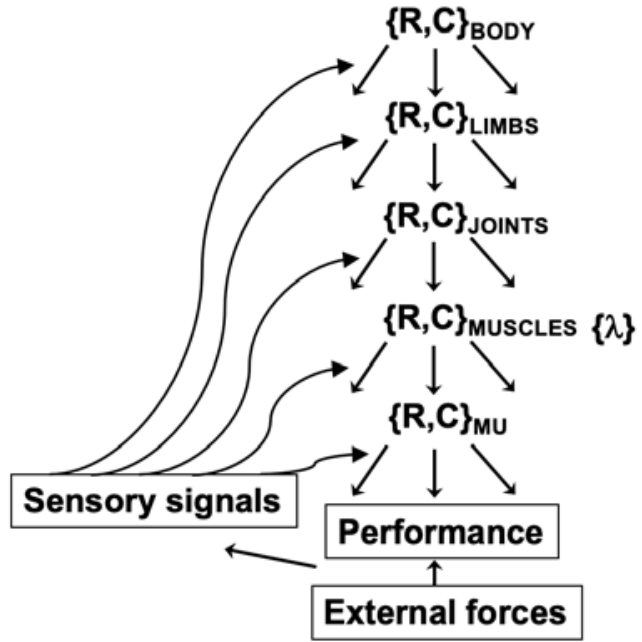

Fig. 2

At the task level, the control of action can be described with a low-dimensional set of $R$ and $C$ commands. Further, a sequence of few-to-many transformations leads to the emergence of the $R$ and $C$ commands at the hierarchically lower levels corresponding to the involvement of joints, muscles, and motor units (MUs).

which can be viewed as a law of nature valid across all skeletal muscles. This law links the two salient variables for the muscle, its length and force (consider only static conditions, for simplicity), with the help of a single parameter $\lambda$ : $\mathrm{F}=f(\mathrm{~L}-\lambda)$, when $\mathrm{L}>\lambda$. Since muscles always act against an external load, the system "muscle + reflexes + load" will come to an equilibrium at a point with certain values of $\mathrm{L}$ and $\mathrm{F}$, when muscle force exactly balances the load (equilibrium point, EP).

If $\lambda=$ const., movements can happen as a result of changes in the external load, for example from $\mathrm{EP}_{0}$ to $\mathrm{EP}_{1}$ in Figure 1A. The production of voluntary movements is associated with changes in $\lambda$, for example from $\lambda_{1}$ to $\lambda_{2}$ in Figure 1B. Note that the same change of $\lambda$ can lead to different peripheral consequences, depending on the external load: From force change in isometric conditions, to movement in isotonic conditions, and to changes in both $\mathrm{F}$ and $\mathrm{L}$ when the muscle acts against a more realistic load. In neurophysiological terms, $\lambda$ has been associated with subthreshold depolarization of the corresponding alphamotoneuronal pool. As a result, $\lambda$ is a neurophysiological variable expressed in millivolts, which is converted by the stretch reflex mechanism into a mechanical variable expressed in units of muscle length (meters). Note that $\lambda$ may be viewed as muscle RC because, if the external load is removed, the muscle will change its length until $\mathrm{L}=\lambda$.

The scheme of control with $\lambda$ can be extended both inside the muscle (to control of individual motor units) and to multi-muscle effectors. Figure 1C illustrates the control of a few motor units (MUs) with the corresponding $\lambda$ values; for a given MU, its firing frequency $\left(f_{\mathrm{MU}}\right)$ increases with mus- cle length leading to an increase in the contribution of that MU to muscle force. Note that muscle $\lambda$ equals the smallest of MU $\lambda s$, and muscle active force is the sum of the contributions of all the MUs.

Any effector in the human body is controlled with opposing muscle groups, agonists and antagonists. The control of an effector can be described with time-varying $\lambda_{\mathrm{AG}}$ and $\lambda_{\text {ANT }}$ (the subscripts stay for agonists and antagonists, Fig. 1D). Alternatively, these two variables can be replaced by an equivalent pair, $R$-command (reciprocal command) and $C$-command (coactivation command). The $R$-command defines a value of the coordinate (e.g., joint angle) where the resultant force variable (moment of force, $\mathrm{M}$, for a joint) is zero. The $C$-command defines the spatial range where the two opposing muscle groups are activated simultaneously. Changing the $C$-command effectively leads to changes in the apparent stiffness ${ }^{16,17)}$ of the effector. So, the control of any effector, up to the whole body may be viewed as setting the $\{R ; C\}$ pair at the task-specific level. For example, for an arm reaching movement, $R$ and $C$ are set for the endpoint of the arm. Then, a sequence of few-tomany transformations (Fig. 2) leads to the emergence of $\{R ; C\}$ pairs at the hierarchically lower levels corresponding to the involvement of joints, muscles, and MUs. Note that all natural movements involve abundant sets of elements: More joints, muscles and motor units as compared to the number of task-related constraints. This offers an exciting opportunity to organize the transformations illustrated in Figure 2 in a way that leads to stabilization of salient, task-specific variables (this issue is described in more detail in the next section).

The idea of muscle control with changes in $\lambda$ has been developed to analyze movements in certain groups of neurological patients, in particular those suffering from spasticity $^{18,19)}$. Healthy humans can relax their muscles within their whole anatomical range and also produce high levels of muscle force also within the whole range. This is illustrated in Figure 3A. Note that relaxing a muscle implies moving its $\lambda$ beyond the longest possible value of muscle length. Producing a very large force with a short muscle requires moving $\lambda$ beyond the anatomical range in the opposite direction. The whole healthy range of $\lambda$ changes is shown as $\lambda_{\text {MIN }}$ to $\lambda_{\text {MAX. }}$.

Patients with spasticity typically display two groups of signs: An inability to activate the affected muscle voluntarily (paresis) combined with unintentional muscle activation leading to spasms and high resistance to external motion (imprecisely addressed as increased muscle tone). The two groups of signs can show parallel changes with treatment, as shown for example in studies of the effects of intrathecal baclofen ${ }^{20)}$. Figure $3 \mathrm{~B}$ illustrates that both paresis and spasms can originate from a single cause: Lost ability to shift $\lambda$ within its whole range. Indeed, if a patient cannot shift $\lambda$ to the left of $\lambda_{\min }$, no active muscle force can be pro- 


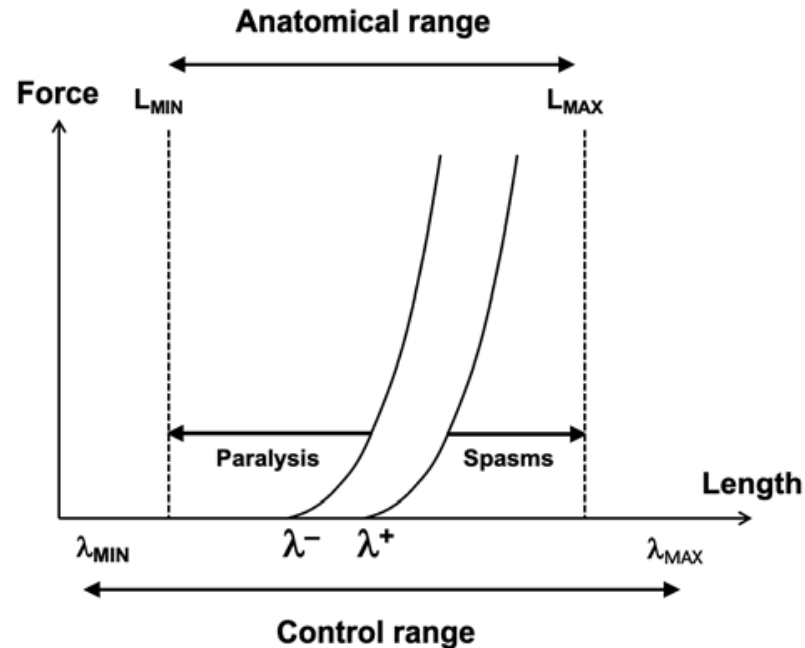

Fig. 3

Healthy humans can shift $\lambda$ over a control range larger than the anatomical range of muscle length. This allows relaxing the muscle within its anatomical range and also producing high levels of muscle force within this range. Spasticity is caused by loss of the ability to shift $\lambda$ within its whole range, e.g., only within $\lambda_{\min }$ to $\lambda_{\max }$. No active muscle force can be produced (paralysis) for the muscle when its length $\mathrm{L}<\lambda_{\min }$. The muscle cannot relax (spasm) when $\mathrm{L}>\lambda_{\max }$.

duced for the muscle when its length $L<\lambda_{\text {min. }}$. If the same patient cannot shift $\lambda$ to the right of $\lambda_{\max }$, the muscle will be unable to relax when its length $\mathrm{L}>\lambda_{\max }$. Recent studies have shown that, indeed, stroke survivors with spasticity show a reduction in the range of voluntary $\lambda$ changes and this reduction correlate with the clinical severity of their $\operatorname{disorder}^{21,22)}$.

\section{Motor Variability: A Crucial Feature of Healthy Movements}

Before moving to the topic of synergies, we have to consider briefly the phenomenon of motor variability, which has been studied at least since the end of the XIXth century. Currently, motor variability is viewed not simply as a result of "noise" within the body but as a crucial, functionally important feature of biological movements ${ }^{23)}$. Indeed, a number of studies have shown that elevated motor variability at the level of involved elements is typical of highly-experienced workers, whereas low motor variability is a predictor of chronic pain emergence ${ }^{24)}$. Along similar lines, very low postural sway may be seen in patients with advanced-stage Parkinson's disease ${ }^{25)}$ who display seriously compromised ability to maintain vertical posture.

The current understanding of motor variability can be traced back to the seminal study by Nikolai Bernstein of hammering by professional blacksmiths. These subjects were arguably best trained to perform the movement of hitting the chisel held by the non-dominant hand by the ham- mer moved by the dominant hand. So, if there was an optimal solution to problems associated with performing this task, Bernstein's subjects were the ones to have it. Nevertheless, Bernstein reported high motor variability at the level of trajectories of both individual joints and the tip of the hammer. His most surprising observation was that the variability of the tip of the hammer was much lower compared to the variability of individual joint trajectories.

Obviously, the brain could not send signals to the hammer, only to muscles that produced joint rotations. How come that the signals to muscles produced highly variable trajectories of the joints, whereas the trajectory of the hammer produced by those joint rotations was much more reproducible? Bernstein introduced the notion of a kinematic synergy stabilizing the hammer trajectory by covarying joint trajectories, an insight that waited for over 50 years to become the foundation of the uncontrolled manifold (UCM) hypothesis ${ }^{26)}$.

\section{Synergies: Ensuring Dynamical Stability of Action}

Bernstein $^{3)}$ emphasized two main functions of the "Level of Synergies" in his multi-level hierarchical system for the control of movements. First, this level was responsible for uniting numerous elements (e.g., muscles) into groups thus alleviating the problem of motor redundancy (reducing the number of degrees-of-freedom) and facilitating control. Second, this level was responsible for ensuring dynamical stability of movements, which is crucial given the unpredictably varying external forces and intrinsic body states.

The first feature of synergies has received much attention recently with various matrix factorization techniques used to identify stable groups of elements, including principal component analysis, factor analysis, non-negative matrix factorization, etc. ${ }^{27,28}$. Bernstein thought of synergies organized in multi-muscle spaces, and this insight has been developed in future studies ${ }^{29-31)}$. This idea has also been applied to both multi-muscle systems, such as those involved in moving individual joints ${ }^{32)}$ and intra-muscle systems such as muscle compartments viewed as constellations of motor units as elements ${ }^{33}$. We would like to emphasize that the idea of grouping elements is far from trivial. Indeed, imagine that a person co-contracts muscles acting at the major joints of a limb. This can be viewed as the means of reducing the number of effective kinematic degrees-of-freedom. But obviously this strategy would increase muscle activation levels and the number of recruited motor units thus making the problem worse at those levels of analysis.

The other feature of synergies is of obvious importance for everyday movements. For example, while walking, we commonly step on small pebbles or other uneven parts of the road. Given the inherently unstable bipedal pos- 

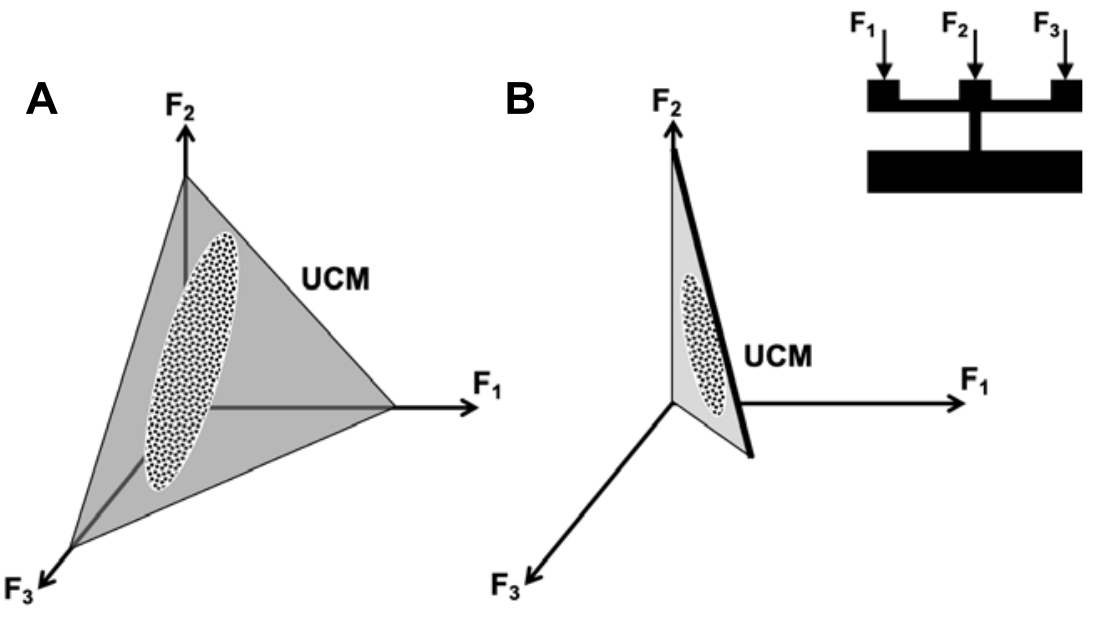

Fig. 4

An illustration of a three-finger task pressing on a rigid surface (the insert). A: The solution space (uncontrolled manifold, UCM, gray) for accurate force production. Data points across repetitive trials are expected to be constrained primarily to the UCM. B: If the task is to produce a certain magnitude of the total moment of force with respect to the pivot, the UCM changes. Both tasks can be accomplished simultaneously: the UCM becomes one-dimensional (the thick line in panel B).

ture, the importance of dynamical stability of walking patterns is obvious. This is also true for other everyday tasks. Bernstein assumed that, in the processes of both evolution and personal experience, the central nervous system elaborates dynamically stable patterns of movements, which do not require continuous corrections by the brain. Note also that, depending on the task, humans stabilize different performance variables, even when those are produced by the same sets of effectors. For example, the human hand can stabilize the resultant force and/or moment of force on the hand-held object. The concept of task-specific stability has formed the foundation of the UCM hypothesis ${ }^{12,34)}$.

According to the UCM hypothesis, the controller acts in a multi-dimensional space of elemental variables and stabilizes a salient performance variables to which all the elemental variables contribute. Consider tasks performed by three effectors (e.g., fingers) pressing in parallel on a rigid surface (the insert in Fig. 4). If the task is to produce a certain magnitude of total force, the solution space is shown in panel A of Figure 4. One can expect data points across repetitive trials to be constrained primarily to the solution space (the UCM) for this task shown as the gray triangle. If the same system is required to produce a certain magnitude of the total moment of force with respect to a pivot (e.g., located under effector E2), the solutions space changes, and the inter-trial data distribution is expected to be constrained to another planar UCM shown in panel B of Figure 4. Note that both tasks can be accomplished simultaneously. Then, the UCM becomes one-dimensional: It is a line formed by the intersection of the two planes (the thick line in panel B).

The illustration in Figure 4 suggests that inter-trial variance can be analyzed to produce an index of stability.
Indeed, stabilization of a salient variable implies higher variance within the corresponding UCM as compared to the orthogonal to the UCM space (ORT). If both indices are quantified per dimension in the corresponding spaces, $V_{\mathrm{UCM}}$ $>V_{\mathrm{ORT}}$ is expected. One can use an index reflecting the normalized difference between $V_{\mathrm{UCM}}$ and $V_{\mathrm{ORT}}$ and use it as an index of a synergy stabilizing the selected performance variable: $\Delta V=\left(V_{\mathrm{UCM}}-V_{\mathrm{ORT}}\right) / V_{\text {Tот }}$, where $V_{\text {Tот }}$ stands for total variance.

Let us emphasize a few important features of the UCM concept. First, low variability of performance does not necessarily imply a strong synergy stabilizing this performance. Performance may be stereotypical (very low $V_{\text {тот }}$ ) without a difference between $V_{\mathrm{UCM}}$ and $V_{\mathrm{ORT}}$ or even with $V_{\mathrm{UCM}}<V_{\mathrm{ORT}}$. Such behavior may be accurate in perfectly predictable conditions in the absence of unexpected perturbations, but it is expected to show major deterioration as soon as conditions become more natural and less predictable. Second, while performance accuracy, by definition depends only on $V_{\mathrm{ORT}}$ but not on $V_{\mathrm{UCM}}$, large $V_{\mathrm{UCM}}$ is important: It reflects channeling effects of unexpected perturbations into the UCM where deviations of elemental variables do not affect the salient performance variable. For example, when you walk down the hallway with a cup of coffee in the hand, you would like the cup to be close to vertical at all times. The cup orientation depends on many factors including all the joint angles. Note that every step is associated with large varying forces between the foot and the floor, which are transmitted to all body parts and perturb all the joints. We can also move the cup with respect to the body while walking without spilling its contents. This is achieved by organizing the relevant joints into a multi-joint 
synergy stabilizing the cup vertical orientation. The synergy is associated with low stability along the UCM, which accepts all the joint angle deviations and protects the cup from excessive tilting.

When a person plans to perform a movement associated with a quick change of a performance variable, having a strong synergy stabilizing that variable may be counterproductive: The actor will have to overcome the resistance to a change in the variable by the synergy. In other words, stability and agility compete with each other. The brain has an ability to attenuate synergies in preparation to quick actions. These anticipatory synergy adjustments (ASAs) have two components. The first is observed when a person knows that a quick action may be required, e.g. to a signal or to a perturbation, but no signal and no perturbation emerge. In such conditions, the synergy index is reduced as compared to a similar task performed in conditions when no quick action can happen ${ }^{35-37}$ ). The other component of ASAs is linked to planning a self-paced quick action; it is associated with a drop in the synergy index 300-400 ms prior to then action initiation ${ }^{38,39)}$ While the synergy index, $\Delta V$, can be used as an index of stability, ASA characteristics (their timing and magnitude) can be used as an index of agility $^{40)}$.

The UCM-based method of analysis of stability has been used to quantify synergies in various spaces of elemental variables, kinetic, kinematic, and electromyographic ${ }^{13,16)}$. Recently, the method of analyzing synergies has been developed to spaces of hypothetical control variables, such as the $R$-command and $C$-command introduced earlier ${ }^{41,42)}$. The first studies on multi-finger force production tasks have documented strong performancestabilizing synergies in the $\{R ; C\}$ space, but expanding this method to other tasks has been challenging. This approach has also not been able to quantify ASAs, which slows down its possible application to clinical studies. There has also been progress in applying this method to analysis of synergies in spaces of individual motor units ${ }^{33)}$, but this approach is in its infancy.

Indices of both stability and agility are sensitive to mild impairments of movements. For example, healthy aging is associated with a drop in both $\Delta V$ and ASA indices $^{43,44)}$. Changes in synergy index have been reported under fatigue in healthy persons ${ }^{45)}$ and in healthy welders ${ }^{46)}$ who are at high risk for developing movement disorders resembling parkinsonism due to the accumulation of manganese in the basal ganglia. Synergies can be improved with practice ${ }^{47)}$, which carries an optimistic message to the field of motor rehabilitation.

\section{Impaired Control of Stability in Neurological Patients}

Problems with controlled movement stability are highly prevalent across the spectrum of neurological disorders. They can be classified into two groups. The first is impairment of dynamical stability leading to disruptions of functional movement patterns by variations in the external forces and intrinsic body states. Loss of postural stability in Parkinson's disease (PD) and ataxia in cerebellar disorders may be seen as most obvious examples. The second is impairment in the ability to initiate a movement or to switch from an unsuccessful movement pattern to an alternative one. Freezing in PD may be viewed as an example of this group. In other words, impaired control of stability may involve impaired stability and impaired agility as its components. These can be studied quantitatively using indices of synergies $(\Delta V)$ and of ASAs, respectively.

Both impaired stability and impaired agility have been documented in PD patients ${ }^{40)}$. Both signs can be observed in early-stage PD and even in sub-clinical cases. Indeed, similar changes in both indices have been reported during studies of multi-finger synergies in both hands of PD patients at stage-I (Hoehn and Yahr 1967), which is defined as the stage with PD symptoms limited to one side of the body only ${ }^{48)}$. Along similar lines, impaired synergies and ASAs in whole-body standing tasks have been reported for stageII PD patients ${ }^{49)}$, i.e., when clinical examination fails to detect signs of postural instability. Recent studies of drugnaïve PD patients have confirmed that problems with synergic control are consequences of the disease itself, not of long-term drug exposure ${ }^{50)}$. Note that the reduced ASA may be viewed as a predictor of episodes of freezing. Indeed, if one cannot destabilize vertical posture during standing, one's feet may feel as if glued to the grounds.

Indices of both stability and agility are sensitive to dopamine-replacement drugs ${ }^{51,52)}$. In contrast, a study of the effects of deep-brain stimulation (DBS) applied to the globus pallidus and to the subthalamic nucleus has confirmed an improvement in indices of agility but not of stability ${ }^{53)}$. These observations suggest caution with the DBS because it can lead to more agile movements in a person with poor stability resulting in undesired effects on fall incidence $^{54)}$.

$\mathrm{PD}$ is not the only disorder showing problems with the two aspects of the synergic control. In particular, similar results have been reported in patients with multi-system brain atrophy $^{55)}$ and with multiple sclerosis ${ }^{56)}$. In contrast, observations in stroke survivors are less consistent. These patients have been reported to show minimal, if any, differences in indices of stability (such as $\Delta V$ ), but significantly reduced ASA indices ${ }^{57,58)}$. Taken together, studies of patients point at subcortical loops as potentially crucial elements of both aspects of the synergic control, ensuring proper stability of actions and being able to attenuate stability prior to a planned quick action. 


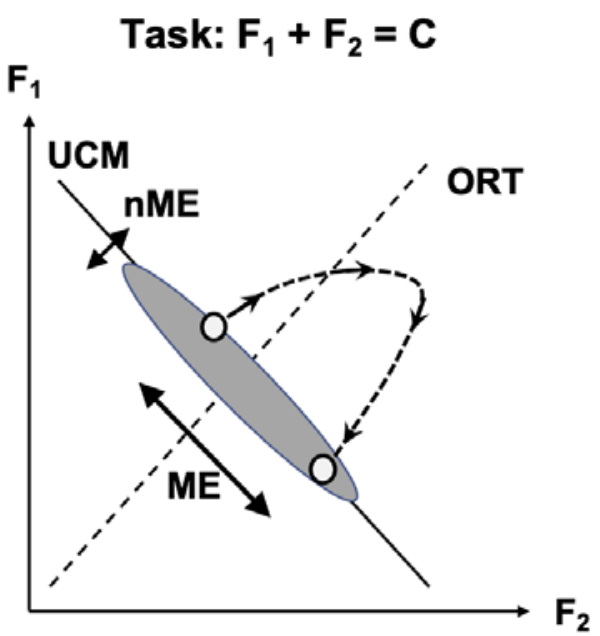

Fig. 5

The task is to produce accurate total force by pressing with two fingers. Larger inter-trial variance along the UCM is expected as compared to that along the orthogonal direction (ORT). If the subject is asked to increase the force quickly and then return to the initial force magnitude, at the end of actions deviations within-the-UCM (motor equivalent, $M E$ ) will be larger than along ORT (non-motor equivalent, $n M E$ ).

\section{Challenges of Clinical Studies}

The reviewed studies suggest that exploring synergic control may be of high clinical importance, in particular for making clinical decisions related to drug therapy, tracking disease progression, and estimating effectiveness of rehabilitation. Quantifying aspects of the synergic control has been truly eye-opening. Broad application of these methods to clinical studies has been slow due to both the conceptual and technical difficulties associated with quantitative studies of action stability and to the necessity to collect multiple trials to compute indices of stability and agility. The former problem can only be solved by education of clinical researchers, in particular by writing reviews targeting clinical professionals, such as this one.

The problem of collecting multiple trials is more challenging. Indeed, patients are less likely to be able to perform a motor task multiple times "in the same way". They typically fatigue faster that healthy persons, and there is limited time they can spend being tested. Indices of synergic control typically show good-to-excellent indices of reliability $^{59)}$, which is a positive sign. On the other hand, to estimate such indices as $V_{\mathrm{UCM}}$ and $V_{\mathrm{ORT}}$ (and indices computed based on those, such as $\Delta V$ and ASA indices), one has to collect a cloud of data points. But how many data points form a "cloud"? This question has been explored recently $^{60,61)}$, and the conclusion is that the minimal number of data points ranges between 12 and 24 .

There have been several attempts to overcome the problem of collecting multiple trials in clinical studies. One of such attempts has been based on the phenomenon of motor-equivalent movement, i.e. movement within the $\mathrm{UCM}^{62,63)}$. For example, if a person performs a task of accurate total force production by pressing with two fingers (Fig. 5), larger inter-trial variance along the UCM is expected reflected in the cloud of data points elongated along the UCM. If this person is now asked to increase the force quickly and then return to the initial force magnitude, at the end of this action, deviations both within-the-UCM (motor equivalent, $M E$ in Fig. 5) and within-the-ORT (non-motor equivalent, $n M E$ ) are expected. Larger deviations along the $\mathrm{UCM}$ are expected, $M E>n M E$ ( similar to $V_{\mathrm{UCM}}>V_{\mathrm{ORT}}$ ). Note that $M E$ and $n M E$ can be measured in individual trials. Estimation of the number of trials needed to obtain reliable estimate of $M E$ and $n M E$ indices has suggested that these indices require half as many trials as needed for reliable estimate of $V_{\mathrm{UCM}}$ and $V_{\mathrm{ORT}}{ }^{59)}$. This is a promising result, but $M E$ and $n M E$ cannot be used to estimate ASAs, which limits their usefulness for clinical studies.

There have also been attempts to improve the situation by using cyclical tasks that allow collecting multiple cycles within a relatively short time ${ }^{33,64)}$. This method, unfortunately, is also unable to quantify ASAs. Combining withina-trial and across-trials analyses has also been used $^{65)}$, but this method has not been developed for clinical studies. A potentially important development has been suggested recently based on bootstrapping techniques that allow overcoming the problem of having relatively few trials and can potentially be used to analyze performance of individual patients $^{611}$.

\section{Implications for Synergic Control in Osteoarthritis}

While most reviewed studies have focused on impaired synergic control in neurological patients, few studies pay attention to that in peripheral disorders such as osteoarthritis (OA). OA is the most common form of arthritis, causing chronic pain and motor impairment ${ }^{66)}$. Earlier studies have shown that OA patients display a reduction in the gray matter in areas related to the transmission of pain, including prefrontal cortex, precentral, and postcentral cortices $^{67-69)}$. Plasticity in the brain has also been observed following the improvements of both pain and the motor function $^{67,70,71)}$. Given plastic brain changes following chronic pain and motor impairment ${ }^{67,70,71)}$, important changes in the synergic control in OA patients can be expected.

The treatments for OA patients have been focused on relieving pain and preventing undesired mechanical stress in the affected joints, which can cause OA progression ${ }^{72,73)}$. Resistance training, mainly for quadriceps muscle strengthening, has been viewed as an effective intervention for improving pain and physical function in patients with knee $\mathrm{OA}^{74,75)}$, and is also recommended as a core treatment in the 


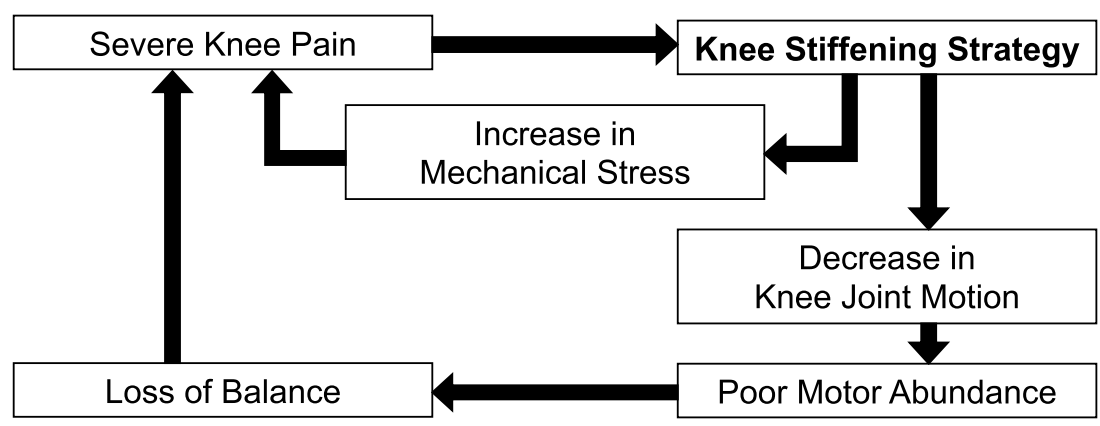

Fig. 6

An illustration of a "vicious circle" due to knee symptom. Knee pain will cause the knee stiffening strategy resulting in the increased mechanical stress at the joint and stronger knee pain. Additionally, the knee stiffening strategy is associated with poor motor abundance, leading to the possibility of the loss of balance (also contributing to knee pain) and, potentially, other comorbidities.

clinical guidelines ${ }^{76)}$. Gait modifications (e.g., the lateral trunk lean walking) have also been used to avoid the pain and undesired mechanical stress ${ }^{77-80}$.

In addition to the pain and undesired mechanical joint stress, impaired gait stability is also a problem for OA patients. OA is viewed as an important risk factor for falls ${ }^{81,82)}$, and an earlier study has reported the greater risk of falls in patients with knee OA, compared to healthy older adults ${ }^{83}$. Given that impaired multi-segmental synergy stabilizing the center of mass (CoM) trajectory during gait has been associated with elevated fall risk $^{84)}$, analysis of the synergic control related to gait stability could be useful for guiding treatment of OA patients. So far, however, few studies have evaluated possible changes in the synergic control in OA.

One study explored the synergic control in patients with knee OA during gait ${ }^{85)}$. These authors evaluated the kinematic synergies controlling CoM trajectory during lateral trunk lean walking, a common gait modification to reduce undesired mechanical stress at the knee ${ }^{85)}$. During walking, real-time visual feedback on the angle of lateral trunk lean was provided, and the patients were instructed to lean the trunk by a certain angle during the stance phase. The lateral trunk lean walking allowed the patients to reduce the undesired knee loading while maintaining the CoM-stabilizing synergy, similar to that during normal walking. These results suggest that the lateral trunk lean walking could be a reasonable treatment strategy for both gait stability and desired knee loading. However, future studies are needed to address such issues as changes in the CoM-stabilizing synergy with OA progression and with long-term treatment, including gait modification.

Gait pattern in patients with knee OA include, in particular, a large reduction in the knee flexion excursion ${ }^{86,87}$. Such stereotypical motor patterns have been viewed as a compensatory strategy to avoid knee pain and reduce the knee joint motion $^{88,89)}$. However, excessive muscle forces required to "stiffen" the knee are expected to increase the me- chanical stress at the joint ${ }^{90,91)}$ leading to a possibility of OA progression and/or increased pain severity. Given the recent results suggesting that muscle co-activation compromises postural stability in young healthy adults ${ }^{92)}$, the "knee stiffening" strategy should probably be discouraged as it might lead to increased incidence of loss of balance and falls.

Note also that an earlier study has explored the effects of joint immobilization, including knee immobilization, on the posture-stabilizing synergy during standing and showed impaired synergic control of posture, especially for tasks with increased complexity ${ }^{93}$. Given that walking may be viewed as a more complex task compared to standing, the "knee stiffening" strategy in patients with knee OA may lead to undesired consequences through the poor use of the kinematic abundance, resulting in elevated fall risk and other pain comorbidities.

To prevent such a "vicious circle" (shown in Fig. 6 as a potential combination of two circles), we have to consider all the salient variables during gait in patients with knee OA. In healthy adults, trajectories of the CoM and swing foot have been viewed as salient performance variables for successful gait ${ }^{84,94)}$. In OA patients who typically show proprioceptive deficits and joint laxity ${ }^{95,96)}$, additional salient performance variables related to the affected joint may need to be stabilized during gait. It is possible that studies of gait in healthy subjects with additional constraints, e.g., reduced knee joint excursion, could provide useful information on possible adaptive gait strategies able to ensure stability of all the salient variables. Such studies would avoid the mentioned "vicious circle", because healthy persons would not experience pain and potentially could explore a broader range of muscle activation patterns. Such studies could provide important insights into treatment strategies of OA directed at optimizing stability of functionally important behaviors such as walking.

Conflict of Interest: The authors have no conflicts of 
interest to disclose.

\section{References}

1) Joseph B: De l'asynergie cerebelleuse. Rev Neurol (Paris). 1899; 7: 806-816

2) Hughlings Jackson J: On the comparative study of disease of the nervous system. Br Med J. 1889; 355-362.

3) Bernstein NA: On the construction of movements, Moscow, Medgiz, 1947. English translation is available in Latash ML (Ed.) Bernstein's construction of movement: original text and commentaries. Routledge, Abingdon, 2020, pp. 1-220.

4) Bernstein NA: The co-ordination and regulation of movements, Published online, Oxford, Pergamon, 1967.

5) Latash ML and Anson JG: What are "normal movements" in atypical populations? Behav Brain Sci. 1996; 19: 55-106.

6) Latash ML and Anson JG: Synergies in health and disease: relations to adaptive changes in motor coordination. Phys Ther. 2006; 86: 1151-1160.

7) Piscitelli D: Neurorehabilitation: bridging neurophysiology and clinical practice. Neurol Sci. 2019; 40: 2209-2211.

8) Latash ML: Physics of biological action and perception. 1st ed, New York, Academic Press, 2019, pp. 1-229.

9) Latash ML: Laws of nature that define biological action and perception. Phys Life Rev. 2021; 36: 47-67.

10) Feldman AG: Referent control of action and perception: challenging conventional theories in behavioral science. New York, Springer, 2015, pp. 1-244.

11) Gelfand IM and Latash ML: On the problem of adequate language in motor control. Motor Control. 1998; 2: 306-313.

12) Scholz JP and Schöner G: The uncontrolled manifold concept: identifying control variables for a functional task. Exp Brain Res. 1999; 126: 289-306.

13) Latash ML: Synergy. New York, Oxford University Press, 2008, pp. 1-412.

14) Feldman AG: Functional tuning of the nervous system with control of movement or maintenance of a steady posture. II. Controllable parameters of the muscle. Biophysics (Oxf). 1966; 11: 565-578.

15) Feldman AG: Once more on the equilibrium-point hypothesis ( $\lambda$ model) for motor control. J Mot Behav. 1986; 18: 17-54.

16) Latash ML and Zatsiorsky VM: Biomechanics and motor control: defining central concepts. New York, Academic Press, 2016, pp. 1-409.

17) Latash ML and Zatsiorsky VM: Joint stiffness: myth or reality? Hum Mov Sci. 1993; 12: 653-692.

18) Levin MF and Feldman AG: The role of stretch reflex threshold regulation in normal and impaired motor control. Brain Res. 1994; 657: 23-30.

19) Jobin A and Levin MF: Regulation of stretch reflex threshold in elbow flexors in children with cerebral palsy: a new measure of spasticity. Dev Med Child Neurol. 2000; 42: 531-540.

20) Latash ML, Penn RD, et al.: Effects of intrathecal baclofen on voluntary motor control in spastic paresis. J Neurosurg. 1990; 72: $388-392$.
21) Turpin NA, Feldman AG, et al.: Stretch-reflex threshold modulation during active elbow movements in post-stroke survivors with spasticity. Clin Neurophysiol. 2017; 128: 1891-1897.

22) Piscitelli D, Turpin NA, et al.: Deficits in corticospinal control of stretch reflex thresholds in stroke: implications for motor impairment. Clin Neurophysiol. 2020; 131: 2067-2078.

23) Newell KM and Corcos DM: Variability in motor control. Illinois, Human Kinetics, 1993, pp. 1-510.

24) Madeleine P, Voigt M, et al.: The size of cycle-to-cycle variability in biomechanical exposure among butchers performing a standardised cutting task. Ergonomics. 2008; 51: 1078-1095.

25) Horak FB, Nutt JG, et al.: Postural inflexibility in parkinsonian subjects. J Neurol Sci. 1992; 111: 46-58.

26) Schoner G: Recent developments and problems in human movement science and their conceptual implications. Ecol Psychol. 1995; 7: 291-314.

27) Ting LH and McKay JL: Neuromechanics of muscle synergies for posture and movement. Curr Opin Neurobiol. 2007; 17: 622628.

28) Tresch MC and Jarc A: The case for and against muscle synergies. Curr Opin Neurobiol. 2009; 19: 601-607.

29) D'Avella A, Saltiel P, et al.: Combinations of muscle synergies in the construction of a natural motor behavior. Nat Neurosci. 2003; 6: 300-308.

30) Krishnamoorthy V, Latash ML, et al.: Muscle synergies during shifts of the center of pressure by standing persons. Exp brain Res. 2003; 152: 281-292.

31) Ivanenko YP, Poppele RE, et al.: Motor control programs and walking. Neuroscientist. 2006; 12: 339-348.

32) Weiss EJ and Flanders M: Muscular and postural synergies of the human hand. J Neurophysiol. 2004; 92: 523-535.

33) Madarshahian S, Letizi J, et al.: Synergic control of a single muscle: The example of flexor digitorum superficialis. J Physiol. 2021; 599: 1261-1279.

34) Latash ML, Scholz JP, et al.: Toward a new theory of motor synergies. Motor Control. 2007; 11: 276-308.

35) de Freitas SMSF, Scholz JP, et al.: Effect of motor planning on use of motor abundance. Neurosci Lett. 2007; 417: 66-71.

36) Tillman $M$ and Ambike S: Cue-induced changes in the stability of finger force-production tasks revealed by the uncontrolled manifold analysis. J Neurophysiol. 2018; 119: 21-32.

37) Tillman $M$ and Ambike $S$ : The influence of recent actions and anticipated actions on the stability of finger forces during a tracking task. Motor Control. 2020; 24: 365-382.

38) Olafsdottir H, Yoshida N, et al.: Anticipatory covariation of finger forces during self-paced and reaction time force production. Neurosci Lett. 2011; 381: 92-96.

39) Shim JK, Olafsdottir H, et al.: The emergence and disappearance of multi-digit synergies during force-production tasks. Exp brain Res. 2005; 164: 260-270.

40) Latash ML and Huang X: Neural control of movement stability: lessons from studies of neurological patients. Neuroscience. 2015; 301: 39-48.

41) Ambike S, Mattos D, et al.: Synergies in the space of control 
variables within the equilibrium-point hypothesis. Neuroscience. 2016; 315: 150-161.

42) Reschechtko S and Latash ML: Stability of hand force production. I. Hand level control variables and multifinger synergies. J Neurophysiol. 2017; 118: 3152-3164.

43) Olafsdottir $\mathrm{H}$, Zhang W, et al.: Age-related changes in multifinger synergies in accurate moment of force production tasks. $\mathrm{J}$ Appl Physiol. 2007; 102: 1490-1501.

44) Olafsdottir H, Kim SW, et al:: Anticipatory synergy adjustments in preparation to self-triggered perturbations in elderly individuals. J Appl Biomech. 2008; 24: 175-179.

45) Singh T, Varadhan SKM, et al.: Fatigue and motor redundancy: adaptive increase in finger force variance in multi-finger tasks. $\mathbf{J}$ Neurophysiol. 2010; 103: 2990-3000.

46) Lewis MM, Lee EY, et al.: Synergy as a new and sensitive marker of basal ganglia dysfunction: a study of asymptomatic welders. Neurotoxicology. 2016; 56: 76-85. doi: 10.1016/j.neur o.2016.06.016.

47) Wu Y-H, Pazin N, et al.: Improving finger coordination in young and elderly persons. Exp Brain Res. 2013; 226: 273-283.

48) Park J, Wu Y-H, et al.: Changes in multifinger interaction and coordination in Parkinson's disease. J Neurophysiol. 2012; 108: 915-924.

49) Falaki A, Huang $X$, et al.: Impaired synergic control of posture in Parkinson's patients without postural instability. Gait Posture. 2016; 44: 209-215.

50) de Freitas PB, Freitas SMSF, et al.: Synergic control of action in levodopa-naïve Parkinson's disease patients: I. Multi-finger interaction and coordination. Exp brain Res. 2020; 238: 229-245.

51) Park J, Lewis MM, et al.: Dopaminergic modulation of motor coordinaton in Parkinson's disease. Parkinsonism Relat Disord. 2014; 20: 64-68.

52) Falaki A, Huang X, et al.: Dopaminergic modulation of multimuscle synergies in postural tasks performed by patients with Parkinson's disease. J Electromyogr Kinesiol. 2017; 33: 20-26.

53) Falaki A, Jo HJ, et al.: Systemic effects of deep brain stimulation on synergic control in Parkinson's disease. Clin Neurophysiol. 2018; 129: 1320-1332.

54) Marconi R, Landi A, et al.: Subthalamic nucleus stimulation in Parkinson's disease. Neurol Sci. 2008; 29 Suppl 5: S389-391.

55) Park J, Lewis MM, et al.: Effects of olivo-ponto-cerebellar atrophy (OPCA) on finger interaction and coordination. Clin Neurophysiol. 2013; 124: 991-998.

56) Jo HJ, Mattos D, et al.: Changes in multidigit synergies and their feed-forward adjustments in multiple sclerosis. J Mot Behav. 2017; 49: 218-228.

57) Reisman DS and Scholz JP: Aspects of joint coordination are preserved during pointing in persons with post-stroke hemiparesis. Brain. 2003; 126: 2510-2527.

58) Jo HJ, Maenza C, et al.: Effects of unilateral stroke on multifinger synergies and their feed-forward adjustments. Neuroscience. 2016; 319: 194-205.

59) de Freitas PB, Freitas SMSF, et al.: Stability of steady hand force production explored across spaces and methods of analy- sis. Exp Brain Res. 2018; 236: 1545-1562.

60) Freitas SMSF, de Freitas PB, et al.: Quantitative analysis of multi-element synergy stabilizing performance: comparison of three methods with respect to their use in clinical studies. Exp Brain Res. 2019; 237: 453-465.

61) Solnik S, Furmanek MP, et al.: Movement quality: a novel biomarker based on principles of neuroscience. Neurorehabil Neural Repair. 2020; 34: 1067-1077.

62) Scholz JP, Schöner G, et al.: Motor equivalent control of the center of mass in response to support surface perturbations. Exp Brain Res. 2007; 180: 163-179.

63) Mattos DJS, Latash ML, et al.: Unpredictable elbow joint perturbation during reaching results in multijoint motor equivalence. $\mathrm{J}$ Neurophysiol. 2011; 106: 1424-1436.

64) Falaki A, Huang X, et al.: Motor equivalence and structure of variance: multi-muscle postural synergies in Parkinson's disease. Exp Brain Res. 2017; 235: 2243-2258.

65) Scholz JP, Kang N, et al.: Uncontrolled manifold analysis of single trials during multi-finger force production by persons with and without Down syndrome. Exp Brain Res. 2003; 153: 45-58.

66) van Baar ME, Assendelft WJ, et al.: Effectiveness of exercise therapy in patients with osteoarthritis of the hip or knee: a systematic review of randomized clinical trials. Arthritis Rheum. 1999; 42: 1361-1369.

67) Rodriguez-Raecke R, Niemeier A, et al.: Structural brain changes in chronic pain reflect probably neither damage nor atrophy. PLoS One. 2013; 8: e54475.

68) Baliki MN, Schnitzer TJ, et al.: Brain morphological signatures for chronic pain. PLoS One. 2011; 6: e26010.

69) Liao X, Mao C, et al.: Brain gray matter alterations in Chinese patients with chronic knee osteoarthritis pain based on voxelbased morphometry. Medicine (Baltimore). 2018; 97: e0145.

70) Liu J, Chen L, et al.: Different exercise modalities relieve pain syndrome in patients with knee osteoarthritis and modulate the dorsolateral prefrontal cortex: a multiple mode MRI study. Brain Behav Immun. 2019; 82: 253-263.

71) Lewis GN, Parker RS, et al.: Structural brain alterations before and after total knee arthroplasty: a longitudinal assessment. Pain Med. 2018; 19: 2166-2176.

72) Tateuchi H: Gait- and postural-alignment-related prognostic factors for hip and knee osteoarthritis: toward the prevention of osteoarthritis progression. Phys Ther Res. 2019; 22: 31-37.

73) Golightly YM, Allen KD, et al.: A comprehensive review of the effectiveness of different exercise programs for patients with osteoarthritis. Phys Sportsmed. 2012; 40: 52-65.

74) Turner MN, Hernandez DO, et al.: The role of resistance training dosing on pain and physical function in individuals with knee osteoarthritis: a systematic review. Sports Health. 2020; 12: 200-206.

75) Zhang Q, Young L, et al.: Network meta-analysis of various nonpharmacological interventions on pain relief in older adults with osteoarthritis. Am J Phys Med Rehabil. 2019; 98: 469-478.

76) Bannuru RR, Osani MC, et al.: OARSI guidelines for the nonsurgical management of knee, hip, and polyarticular osteoarthritis. Osteoarthr Cartil. 2019; 27: 1578-1589. 
77) Hunt MA and Takacs J: Effects of a 10-week toe-out gait modification intervention in people with medial knee osteoarthritis: a pilot, feasibility study. Osteoarthr Cartil. 2014; 22: 904-911.

78) Fregly BJ, Reinbolt JA, et al.: Design of patient-specific gait modifications for knee osteoarthritis rehabilitation. IEEE Trans Biomed Eng. 2007; 54: 1687-1695.

79) Gerbrands TA, Pisters MF, et al.: Lateral trunk lean and medializing the knee as gait strategies for knee osteoarthritis. Gait Posture. 2017; 51: 247-253.

80) Hunt MA, Charlton JM, et al.: Clinical and biomechanical changes following a 4-month toe-out gait modification program for people with medial knee osteoarthritis: a randomized controlled trial. Osteoarthr Cartil. 2018; 26: 903-911.

81) Manlapaz DG, Sole G, et al.: Risk factors for falls in adults with knee osteoarthritis: a systematic review. PM R. 2019; 11: 745757.

82) Foley SJ, Lord SR, et al.: Falls risk is associated with pain and dysfunction but not radiographic osteoarthritis in older adults: tasmanian older adult cohort study. Osteoarthr Cartil. 2006; 14: 533-539.

83) Levinger P, Menz HB, et al.: Physiological risk factors for falls in people with knee osteoarthritis before and early after knee replacement surgery. Knee Surgery, Sport Traumatol Arthrosc. 2011; 19: 1082-1089.

84) Yamagata M, Tateuchi H, et al:: The relation between kinematic synergy to stabilize the center of mass during walking and future fall risks: a 1-year longitudinal study. BMC Geriatr. 2021; 21: 240.

85) Tokuda K, Anan M, et al.: Biomechanical mechanism of lateral trunk lean gait for knee osteoarthritis patients. J Biomech. 2017; 66: 10-17.

86) Mills K, Hunt MA, et al:: Biomechanical deviations during level walking associated with knee osteoarthritis: a systematic review and meta-analysis. Arthritis Care Res. 2013; 65: 1643-1665.
87) Favre J and Jolles BM: Gait analysis of patients with knee osteoarthritis highlights a pathological mechanical pathway and provides a basis for therapeutic interventions. EFORT Open Rev. 2016; 1: 368-374.

88) Astephen JL, Deluzio KJ, et al.: Biomechanical changes at the hip, knee, and ankle joints during gait are associated with knee osteoarthritis severity. J Orthop Res. 2008; 26: 332-341.

89) Heiden TL, Lloyd DG, et al.: Knee joint kinematics, kinetics and muscle co-contraction in knee osteoarthritis patient gait. Clin Biomech (Bristol, Avon). 2009; 24: 833-841.

90) Brandon SCE, Miller RH, et al.: Selective lateral muscle activation in moderate medial knee osteoarthritis subjects does not unload medial knee condyle. J Biomech. 2014; 47: 1409-1415.

91) Adouni M and Shirazi-Adl A: Partitioning of knee joint internal forces in gait is dictated by the knee adduction angle and not by the knee adduction moment. J Biomech. 2014; 47: 1696-1703.

92) Yamagata M, Falaki A, et al.: Effects of voluntary agonistantagonist coactivation on stability of vertical posture. Motor Control. 2019; 23: 304-326.

93) Hsu WL: Adaptive postural control for joint immobilization during multitask performance. PLoS One. 2014; 9: e108667.

94) Krishnan V, Rosenblatt NJ, et al.: The effects of age on stabilization of the mediolateral trajectory of the swing foot. Gait Posture. 2013; 38: 923-928.

95) Lee SS, Kim HJ, et al.: Comparison of proprioception between osteoarthritic and age-matched unaffected knees: a systematic review and meta-analysis. Arch Orthop Trauma Surg. 2021; 141: 355-365.

96) van Tunen JAC, Dell'Isola A, et al.: Association of malalignment, muscular dysfunction, proprioception, laxity and abnormal joint loading with tibiofemoral knee osteoarthritis - a systematic review and meta-analysis. BMC Musculoskelet Disord. 2018; 19: 273. 